\title{
Suporte articulado para o tratamento cirúrgico da escoliose: nota prévia
}

Support for surgical treatment of scoliosis: foreword

\begin{abstract}
Soporte articulado para el tratamiento quirúrgico de la escoliosis: nota previa
\end{abstract}

\section{Francisco Limeira dos Santos Neto*}

\section{RESUMO}

Foi desenvolvido um suporte para ser utilizado nas cirurgias de correção da escoliose que permite a realização de manobras para a correção da deformidade. O objetivo deste relato inicial é a apresentação do conceito do suporte desenvolvido e os resultados iniciais com a sua utilização.

DESCRITORES: : Escoliose/ cirurgia; Dispositivos de fixação ortopédica

\begin{abstract}
One support was developed for use in surgery for correction of scoliosis. This support allows the execution of surgery for the correction of the deformity, and the initial purpose of this report is the presentation of the concept support development and the initial results with its use.
\end{abstract}

KEYWORDS: Scoliosis/surgery; Orthopedic fixation devices

\begin{abstract}
RESUMEN
Fue desarrollado un soporte para ser utilizado en las cirugías de corrección de la escoliosis. El soporte desarrollado permite la realización de maniobras para la corrección de la deformidad y el objetivo de este relato inicial es la presentación del concepto del soporte desarrollado y los resultados iniciales con su utilización.
\end{abstract}

DESCRIPTORES: Escoliosis/ cirugía; Dispositivos de fijación ortopédica

\section{INTRODUÇÃO}

O sucesso da cirurgia na coluna pode estar relacionado com o adequado posicionamento do paciente. Os objetivos do posicionamento trans-operatório em decúbito ventral estão relacionados com a preservação e melhora do alinhamento sagital da coluna vertebral, a descompressão do abdômen; a proteção das eminências ósseas e o acesso ao exame radiológico da coluna vertebral ${ }^{1}$. Relton $\&$ Hall $^{2}$ foram os pioneiros a enfatizar esses aspectos e desenvolveram um suporte cirúrgico para reduzir o sangramento trans-operatório. Goldstein ${ }^{3}$ descreveu a correção pré-operatória com a utilização de gesso com o distrator (turnbuckle cast) e também enfatizou a importância do posicionamento das almofadas axilares para evitar compressão do plexo braquial.

Vários tipos de suporte intra-operatórios foram desenvolvidos: o suporte de Wilson constituído por duas almofadas laterais e paralelas; a mesa de Andrews, que mantém os joelhos e quadris em $90^{\circ}$ graus de flexão; e a mesa de Jackson que permite acesso a toda coluna vertebral pela via posterior1. Dentre os suportes disponíveis não existe nenhum que permita a correção intra-operatória da deformidade ver- tebral, e esse fato foi a motivação para o desenvolvimento do suporte que desenvolvemos.

O objetivo deste relato inicial é apresentar características de suporte para a correção no plano transversal e frontal por meio das manobras realizadas no suporte e os resultados preliminares com a sua utilização.

\section{DESCRIÇÃO DO SUPORTE}

O suporte articulado consiste de duas placas em acrílico na forma de um pentágono irregular, unidas pelos vértices por meio de uma articulação universal, permitindo movimentos laterais e de torção das placas (Figura 1).

A correção pré-operatória facilita a colocação do material cirúrgico com uma melhor visualização radiológica (Figura 2), de modo a dispensar o uso de instrumentais cirúrgicos para a correção da deformidade, e reduzindo a duração do procedimento cirúrgico. A utilização do suporte articulado permite a correção da deformidade (escoliose) antes da aplicação do instrumental e pode dar proteção ao sistema nervoso central e, ainda, evitar a hipercorreção.

\footnotetext{
Trabalho realizado no Departamento de Ortopedia e Traumatologia da Faculdade de Ciências Médicas da Santa Casa de Misericórdia de São Paulo - FCMSCMSP

- São Paulo (SP), Brasil.

*Professor Adjunto do Departamento de Cirurgia do Centro de Ciências da Saúde da Universidade Federal de Pernambuco - UFPE - Recife (PE), Brasil.
} 

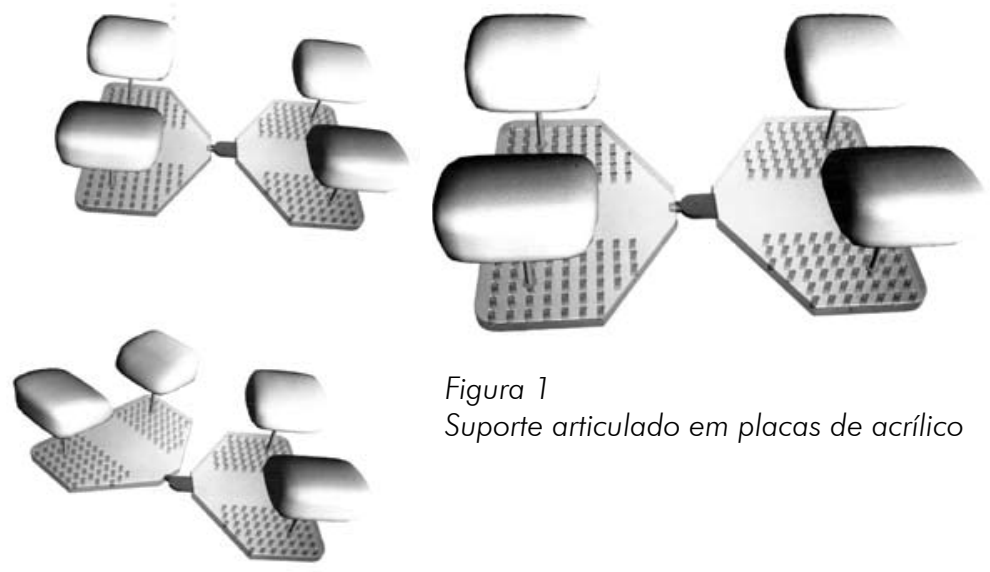

Figura 1

Suporte articulado em placas de acrílico
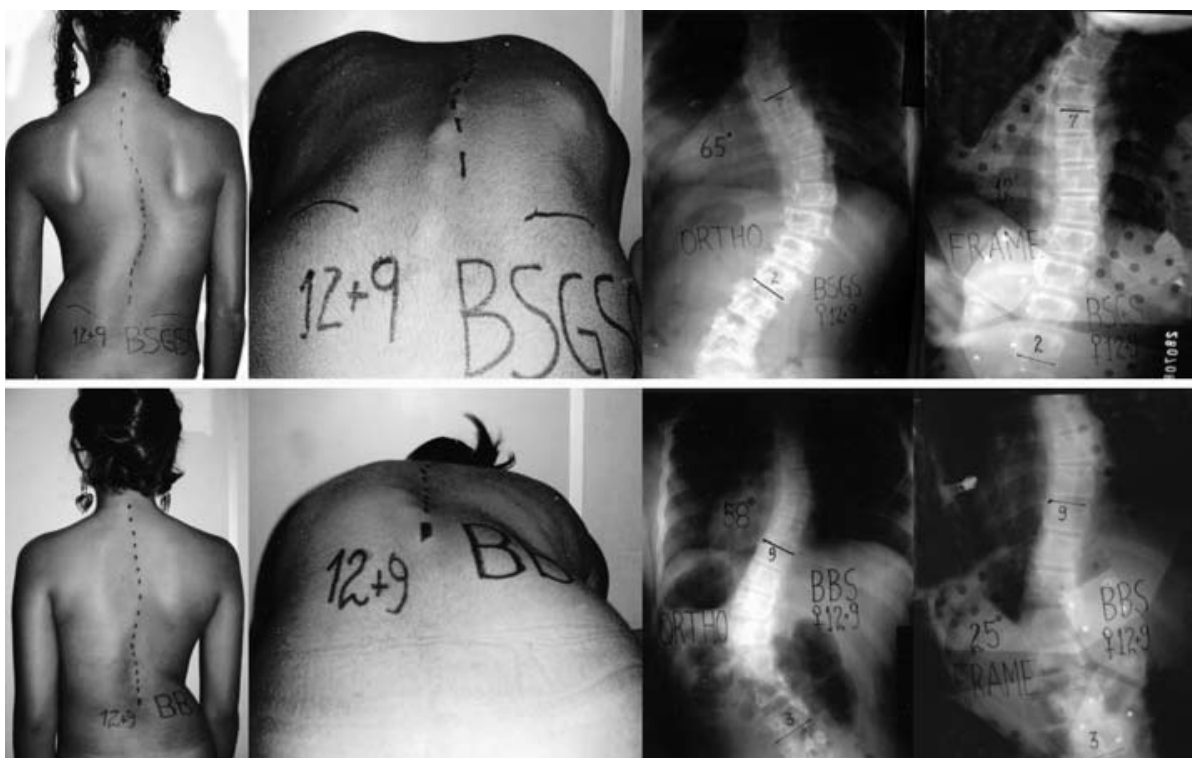

Figura 2

Pacientes nas posições ortostática, inclinação anterior e sobre o suporte com correção da curva

\section{ASPECTOS ÉTICOS}

O presente trabalho foi encaminhado para a Comissão de Ética em Pesquisa do Centro de Ciências da Saúde da Universidade Federal de Pernambuco.

\section{CONCLUSÃO}

O suporte articulado para o tratamento cirúrgico da escoliose é um aparelho experimental e está sendo realizado, no momento, um ensaio clínico controlado com a sua aplicação, para avaliar sua efetiva eficácia.

\section{REFERÊNCIAS}

1. DeWald RL. Spinal deformities: the comprehensive text. New York: Thieme; 2003.

2. Relton JES, Hall JE. An operation frame for spinal fusion. A new apparatus designed to reduce hemorrhage during operation. J Bone Joint Surg Br 1967; 49(2)327-32.

3. Goldstein LA Results in the treatment of scoliosis with turnbuckle plaster cast correction and fusion. J Bone Joint Surg. 1959;41(2):321-35.

\section{Correspondência}

Francisco Limeira dos Santos Neto

R. Bebinho Salgado, 33

CEP: 52071-321

Apipucos - Recife - PE, Brasil 